\title{
Integrating reproductive and child health and HIV services in Tanzania: Implication to policy, systems and services
}

PRINCE P. MUTALEM WA ${ }^{1}$, WILLIAM N. KISINZA ${ }^{2}$, MICHAEL MUNGA ${ }^{1}$, JANESTA A.E. URASSA ${ }^{1}$, STAFFORD KIBONA ${ }^{3}$, UPENDO MWINGIRA ${ }^{1}$, CHRISTINA LASWAY ${ }^{4}$, STELLA KILIMA ${ }^{1}$, FILEMONI TENU ${ }^{2}$, STELLA MUJAYA ${ }^{4}$ and WILLIAM J . KISOKA ${ }^{1}$

'National Institute for Medical Research Headquarters, P o Box 9653, Dar es Salaam, Tanzania

${ }^{2}$ National Institute for Medical Research, Amani Research Centre, P o Box 81, Muheza, Tanzania

${ }^{3}$ National Institute for Medical Research, Tabora Research Centre, P o Box 482, Tabora, Tanzania

${ }^{4}$ Family Health International, P o Box 9292, Dar es Salaam, Tanzania

\begin{abstract}
In Tanzania, reproductive health and HIV services are coordinated by the Ministry of Health and Social Welfare in two separate units namely Reproductive and Child Health Section and the National AIDS Control Programme. The importance of integrating the two services that are vertically run is expected to improve access to and uptake of key essential services and extend coverage to underserved and vulnerable populations and thus minimizing missed opportunities. Experts around the world recognize the central role of Sexual and Reproductive Health (SRH) services in preventing HIV infection. Evidence suggests that improving access to contraception for women to prevent pregnancy is an important and cost-effective way to prevent HIV-positive births. Integrating SRH and HIV services therefore verifies its importance for improving maternal and child health as well as leading to prevention of HIV infection. The primary objective of this review was to gain an understanding of the current linkages between SRH and HIV within Tanzania's policies, programmes, systems and services. Policy documents, guidelines, national laws, and published reports on SRH and HIV were reviewed. The majority of the reviewed documents mentioned fundamentals of integration between SRH and HIV. Majority of policies and guidelines both in family planning (FP) and HIV documents mandate bi-directional linkages. This review suggests that there are linkages between the two services and can be operationalised together. However, policies and guidelines only specify services to be integrated without due consideration of resources and structural orientation for linked services.
\end{abstract}

Keywords: Sexual, Reproductive Health, child health, Family Planning, HIV, Tanzania

\section{Introduction}

Experts around the world increasingly recognize the central role of family planning (FP) in the prevention of HIV infection. Global evidence suggests that among HIV-infected women who do not wish to get pregnant, improving access to contraception is an important and cost-effective way to prevent HIV-positive births and, by extension, the number of children needing HIV treatment, care, and support (Sweat et al., 2004; Stover et al., 2003; Rochat et al., 2006). Indeed, prevention of unintended pregnancies in HIV-positive women is one of the four cornerstones of a comprehensive approach to Preventing Mother-to-Child Transmission (PMTCT) of HIV. Nevertheless, unintended pregnancies among women with HIV remain unacceptably high (Desgrées-du-Loû et al., 2000; Homsy et al., 2009, Martin et al., 1997).

Likewise, for women and men with HIV who want to have children, linkages between reproductive health $(\mathrm{RH})$ and HIV programmes are important to ensure access to services that will allow for a safe pregnancy and delivery, including but not limited to pre-conception counseling and antiretroviral therapy (ART) to reduce vertical transmission risks. Because both closely spaced births and HIV/AIDS increase risks of adverse pregnancy outcomes - such as low birth weight, preterm birth, and infant mortality - counseling on healthy timing and spacing of pregnancies is especially important for women with HIV who wish to have a child (Brocklehurst et al., 1998; Abrams et al., 1995; M oHW, 2008).

Often, family planning and HIV/AIDS programmes are designed and implemented separately. However, clients of these programmes have multiple, related sexual and reproductive health needs that integrated services could potentially cater them more effectively. While some need sexual and reproductive health (SRH) services because they are sexually active, of 
reproductive age, and want to prevent pregnancy, others wish only to learn their HIV status, oblivious of the risks carried by unintended pregnancies. Furthermore, with FP and HIVIAIDS services traditionally being offered separately, the benefits for integrating the two programmes are lost. For instance, whilst much attention is given to preventing HIV in infants once a pregnant woman knows she is infected with HIV, through promotion of testing and counseling among pregnant women and treatment of infants during birth, integration of services enables women with known HIV+status to be reached earlier to prevent a future unintended pregnancy. In turn, a cost- and time-saving of financial and human resources can be realized. Furthermore, integration of services can reduce the probability of missing opportunities to engage HIV/AIDS clients to consider family planning services. Regular repeat client visits in care and treatment sites allow for repeated message in circumstances where client fertility desires are changing.

The government of Tanzania recognizes the need for an informed, nuanced and comprehensive response to strengthening and improving the linkages between these services. Beginning with one component of SRH and HIV/AIDS integration (FP and HIV), the Ministry of Health and Social Welfare with support from the USAID hosted a Stakeholder Consultative Meeting on Integrating Family Planning and HIV/AIDS Services in Tanzania on 2008. Some of the recommendations from the meeting included the need to translate policies into actions; an analysis of potential benefits and challenges of pursuing integration; revising and updating current training materials and the national curricula; the need for integrated services to minimize missed opportunities; strengthening facility based services and community based models of integration; and, most importantly, the development of a National Strategic Framework for Family Planning/HIV Integration in Tanzania (M oHSW, 2008).

Integrating FP services into rapidly expanding HIV prevention and care programs can increase access to contraceptive methods. Likew ise, integrating HIV services into FP programmes can maximize the reach of HIV testing, as well as of care and treatment and such synergies can dramatically enhance the public health impact of the HIV and FP programs and services (MoHSW, 2008). There is international consensus around the need for effective linkages between responses to HIV and SRH including recommendations for specific actions at the levels of policy, systems, and services (WHO, 2004). Much remains unknown about which linkages have the greatest impact, and how best to strengthen selected linkages in different programme settings. Moreover, stronger bi-directional linkages between SRH and HIV-related programmes could lead to a number of important public health, socioeconomic and individual benefits.

The objective of this review was to analyse the current status of SRH and HIV linkages at policy, program and delivery levels. The specific objectives were: (i) to assess the current linkages between SRH with a focus on FP and HIV within Tanzania's policies, systems and services; (ii) to identify current critical gaps in the policy and programmatic environments to support effective and sustainable linkages in service delivery; and (iii) to establish a foundation for the development of a National Strategic Framew ork for FP/HIV Integration in Tanzania.

\section{Materials and Methods}

The review was done manually after obtaining documents related to SRH and HIV services from published articles, surveys, research, national plans, guidelines and policy documents and other written reports. A total of $41 \mathrm{HIV}$ and SRH related policy and guideline documents were included in the review process. A careful collection and review of these documents provided overall introductory insights and facts on the state of linkages in SRH and HIV policies, systems and service delivery in the country. This review was guided by the key words that included HIV, AIDS, and Sexual Reproductive Health, services integration, linkages and Tanzania.

A review of insights and facts on the state of linkages in policies, guidelines and laws was done. The review was based on four coordinated service areas (a) support for integration in national health and development policies and guidelines; (b) support for integration in Sexual 
and Reproductive Health (SRH) policies and guidelines; (c) support for integration in HIV policies and guidelines and;(d) service accessibility according to national laws, policies and guidelines.

\section{Policy analysis of opportunities and gaps in linkages between SRH and HIV services}

Although several key health policies and guidelines in Tanzania call for the integration of FP and HIV services, in practice these mandates have not been fully realized. The Tanzania Health Policy (Sera ya Afya, 2007) aims at providing direction towards improvement and sustainability of the health status of all the people, by reducing disability, morbidity and mortality, improving nutritional status and raising life expectancy. The findings of the analysis acknowledge the existence of Essential Health Care Package which advocates an integrated collection of cost effective interventions which address the main health services including HIV and SRH. Showing the importance of services integration it has been stated that integrating disease control programmes could gradually minimize the costs for utilizing the available resources and at the other hand maximizing the benefits to be attained (HRHS, 2008).

The National Standards for Adolescent Friendly Reproductive Health Services (2005) outlines the minimum standards of care that should guide the delivery of quality adolescent friendly reproductive health services in the country. On its advocacy to services integration, the document has explicitly stated that "if well integrated the proposed range of adolescent reproductive health services to be provided is in tandem with Essential Reproductive Health Package of the Ministry of Health".

The Health Sector Strategic III of 2009-2015 contributes to Tanzania's efforts to reduce child and maternal mortality and to control important infectious diseases. On page 52 it advocates the integration with the view that such integration will improve the development of targeted action plans, assisting the poorest and most vulnerable. It also suggests that the work of the Social Welfare Department and the work of health program e.g. HIV/AIDS, TB, Malaria, Environmental Health, Maternal and Child Health could work together with the main aim of alleviating the diseases (MoHSW, 2009).

The Plan III Human Resources for Health Strategic Plan, 2008-2013 (URT/M oHSW, 2008) guides the Health and Social Welfare sector in the proper planning, development, management and utilization of the most important resource of all which is the human resource. Although it does not mention explicitly linking HIV and SRH services it recognizes the pressing health and social welfare challenges that Tanzania faces including HIV/AIDS and high maternal mortality rate. The document described the importance of having a strategic plan that guides the systematic and proactive implementation of human resource initiatives. The plan pledges to assist the country in achieving the right number of health and social welfare workers, with the requisite knowledge and skills that are effectively managed and are equitably distributed to ensure that national health goals are attained. The plan hence desires to contribute to the improvement of human resource financing by providing comprehensive budgets and identifying ways of mobilizing adequate resources from all health stakeholders.

The National Strategy for Growth and Reduction of Poverty (NSGRP) (URT, 2010) is the national organizing framework for putting the focus on poverty reduction within the country's development agenda. The NSGRP keeps in focus the aspirations of Tanzania's Development Vision (URT, 1999) for high and shared growth, high quality livelihood, peace, stability and unity, good governance, high quality education and international competitiveness. The document addresses existing barriers and increasing access to high quality care particularly for women and children in the rural areas. This policy document has given a high priority of services integration. In its verses, it has included family planning, adolescent reproductive health, antenatal care, emergency obstetric care, post-natal care and newborn care. The NSGRP identifies specific measures (e.g. access to family planning services, improved maternal health care) to address gender inequalities and address the increasing HIV infection among women. 


\section{Support for integration in Sexual and Reproductive Health policies and guidelines}

The National Policy Guidelines for Reproductive and Child Health Services (URT/MoH, 2003) was developed by the Ministry of Health to give directions and facilitate effective implementation of Reproductive and Child Health interventions. The guidelines mandate the integration of HIV and AIDS services into family planning and reproductive health in the context of provision of health education. The document has stipulated that all persons shall have the right to information, education and communication on issues relating to sexually transmitted infections (STIS), HIV and AIDS. It envisages that government shall have to facilitate public, voluntary agencies, nongovernmental organizations (NGOs) and private institutions incorporate STIs, HIV and AIDS issues in the curricula for young people in and out of school settings. Furthermore, tailoring education and counselling for STIs, HIV and AIDS where there shall be special education and counselling services on STIS, HIV and AIDS tailored for specific population segment.

The guidelines also mandate integration under dual protection and provision of services. It is clarified that the M inistry of Health and Social Welfare through Tanzania Bureau of Standards shall ensure availability of quality condoms for dual protection. Although what is written is not fully articulated, the document further insists that all reproductive and child health interventions in public, private NGOs and voluntary sector shall integrate STIS, HIV and AIDS prevention and care and also that all screening and HIV testing shall be done in accordance with Ministry of Health and Social Welfare guidelines and standards whereas all Reproductive and Child Health providers in public, private, NGOs and voluntary agencies shall adhere to these guidelines as well as retaining at the highest level confidentiality protocols on STIS, HIV and AIDS screening and testing. The policy guidelines mandate that "Reproductive health programmes should incorporate HIV and AIDS services within Information, Education and Communication, counselling, dual protection recommendations, STI management, screening and testing, and Prevention of Mother to Child Treatment.

The National Road Map Strategic Plan to Accelerate Reduction of Maternal, Newborn and Child Deaths in Tanzania 2008 - 2015, plans to reduce maternal, newborn and child mortality in line with the tenets of the New Delhi Declaration 2005 (MoHSW, 2008). Tanzania and other countries committed to develop one national Maternal, Neonatal and Child Health (MNCH) plan for accelerating the reduction of maternal, newborn and child deaths, in order to improve coordination, align resources and standardize monitoring. Integration of HIV and AIDS and Sexual and Reproductive Health services is explicitly emphasized in this document. Showing the reached level of integration by September 2007, there were about 1,311PMTCT sites established within Reproductive and Child Health clinics throughout the country (MoHSW, 208). Furthermore, the evidence of services integration is exemplified by the current integration of services of PMTCT, Ante-natal care, nutrition, Integrated Management of Childhood Illnesses (IMCl) and other HIV/AIDS services. This has enhanced opportunities for reducing paediatric HIV and its associated deaths (MoHSW, 2008).

The Comprehensive Family Planning Procedure Manual (URT/MoHSW 2007) guides on how to perform procedures or communicate with clients. It provides procedures that can be fully performed as they are and that can be selectively performed. The manual states of "the need to continue with efforts of providing as many services to clients in one health facility (integrating other RCH services to family planning"). According to the document, educating and counselling FP/RCH Clients includes planning and maternal health, voluntary counselling and testing for HIV. It foresees the need of promoting reproductive health of men and men's participation in their partners' health issues, the need for FP providers to contribute to the control of STI, HIV and AIDS and promotion of provider sensitivity to adolescents' needs which is by actual fact integration of the two services. Among several integrated activities of HIV and SRH services which are advocated in the manual are on the risk of STI, HIV and AIDS during counselling on modern FP methods, and counselling on risk of HIV in the procedures for post-operative care 
after tubal ligation. Tanzania aims at sustaining the quality of family planning and other reproductive services which are in response to several interventions that are in line with national guidelines of reproductive and child health. The document stipulate that "the services provider should provide other RCH services to clients and these include STI, HIV and AIDS services, postpartum care, infertility care, nutrition care and cancer screening, they should as well refer clients to other health, social and community services as necessary". The document also states that a criterion for providing integrated services: "The service provider educates, counsels, and manages for: ... STI, HIV and AIDS" Although HIV is not specifically mentioned, referrals are recommended within the package of components and standards for 'Integrated Services'. Although HIV is not specifically named in the Training Standards, the "Approved Ministry of Health curricula" includes "Integrated RCH" which may or may not include HIV and AIDS training (MoHSW; 2005).

The Ministry of Health and Social Welfare in Tanzania has listed HIV/AIDS among the reproductive health problems facing youth and services recommended include voluntary counselling and testing (VCT), management of STIS, HIVIAIDS, and referrals (although generically stated, this most likely includes referrals for HIV services). Those services should include prevention, promotion, and curative and rehabilitation activities and should include among others, testing services, management of STI, condom provision and promotion and other related services (M oHSW, 2005).

\section{Support for integration in HIV/AIDS policies and guidelines}

National Policy on HIV/AIDS (URT/PMO 2001) provides the framework, direction and general principles in the national response interventions in the prevention, care and support of those infected and affected by the epidemic and mitigation of its impact. On HIV and Reproductive and Sexual Health services integration, the policy has stated that, HIV/AIDS information should be introduced early enough so as to protect the children who are not yet sexually active and that reproductive and sexual health should be incorporated in the school curricula.

The plan in the policy is to collaborate with Local Government Councils, NGOs and Faithbased Organizations (FBOs) shall develop participatory HIV/AIDS, sexual and reproductive health education programmes for the out of school youths; Community programs shall address the issues of multiple sex partnership and the issues of gender and reproductive rights in relation to the spread and transmission of HIV/AIDS (URT/PMO 2001). It further recommends reproductive health and HIV curricula be included for schools and developed for out-of-school youth and counselling and provision of family planning be included in the PM TCT section. It further gives a picture that integrated, quality and user-friendly reproductive health services shall be made accessible to men, women and the youth" which is itself an acceptance of services integration.

According to the Second National Multi-Sectoral Strategic Framework on HIV and AIDS, there are guidelines which have several strategies aiming at reducing the transmission of HIV from mothers to their children, during pregnancy, birth and/or breast-feeding and ensures entry into care and treatment for mother and baby. On services linkages the guidelines have focused on promoting access to family planning integrated in reproductive and child health to prevent unwanted pregnancies in HIV positive women. It further opts on the expansion of PMTCT services through integration of testing and counselling with opt-out option in antenatal care services. Elsewhere, it advocates the distribution of condoms to sexually active population, especially in the rural areas, who use condoms consistently and correctly mainly for dual protection (PM O/TACAIDS, 2003).

The Framework includes several opportunities to address linkages between HIV/AIDS and family planning policies, practices, and services by recommending and recognizing the special needs of specific groups such as women and youths and giving evidence where mother to child transmission (MTCT) represents $18 \%$ of all new infections each year. Elsewhere in the document, 
there are brief mentions of linking HIV testing and counselling to existing reproductive and child health services, FP as part of PMTCT strategy, and condoms representing dual protection for women. How ever, the strong emphasis on family planning or the inclusion of any FP indictors is absent (PMO/TACAIDS, 2003). Nonetheless, the document has shown that there are missed opportunities to highlight the synergies between HIVIAIDS and family planning.

The hallmark of the Health Sector Strategy for HIV/AIDS, 2008-2012 (HSHSP, 2008) is the move towards integrating HIV/AIDS in the functions of all the structures of the M inistry of Health and Social Welfare and all the institutions in the health sector. It clearly accepts that the role that could maximize the missed opportunities when services are integrated. In it the role of the National AIDS Control Programme (NACP) has been redefined to become a specialized agency of the Ministry of Health which manages this transition from a largely vertical programme to an integrated function of the health sector. In highlighting a need of linkage of SRH and HIV services specifically, the document maintains that "integration of PMTCT interventions in ANC, nutrition programmes, IMCl and other HIV/AIDS services enhances opportunities for reducing paediatric HIV and its associated death"

The National Prevention of Mother to Child Transmission Guidelines (URT/MoHSW 2007) was developed intentionally in order to equip health workers with knowledge in order to have a uniform approach in carrying out PMTCT interventions in the Reproductive and Child Health services. The guidelines recognize the importance of services linkages by stating that "facilities offering PMTCT services should also cater for special family planning services and that in remote areas, outreach VCT and PMTCT should be integrated in the existing MCH services". The guidelines use the four UN/WHO elements of a comprehensive PMTCT approach as cornerstones, including the prevention of unintended pregnancies among women infected with HIV. On HIV and SRH services integration, the guideline, has pinpointed family planning to be part of a comprehensive public health strategy to prevent MTCT. All HIV-infected women and their partners should receive family planning and counselling and should be provided with access to effective contraceptive methods. Furthermore, family planning counselling and provision is recommended within the guidelines for post-test counselling and postpartum education and services and it is included in the 'Essential package of integrated ANC services for HIV-infected women', and as part of 'Comprehensive care, treatment, and support for mothers and families with HIV infection'. The guidelines recommend that PMTCT programmes institute an integrated logistics system, and orders for supplies should include family planning commodities.

Although one of the nine key PM TCT M onitoring Indicators is "Percent of postpartum HIVinfected women who receive family planning services", there is no reference to family planning counselling or provision on the Monthly Summary Forms for the PMTCT Antenatal Clinic, the Mother and Child M onthly Follow Up, or the PMTCT Care Register (URT/M oHSW, 2007). There is a column to mark which family planning method is currently being used by a client on the Mother and Child Follow-up Register, but columns to indicate FP needs or counselling provided are missing. To monitor this key indicator, family planning information needs to be included in the standardized forms and registers.

The National Guidelines for Management of HIV and AIDS (NACP, 2008) assists providers in managing people living with HIV/AIDS. The document acknowledges FP as part of a comprehensive package of prevention interventions to be offered to people living with HIV/AIDS. The document states that "Quality PMTCT services should be integrated within Reproductive and Child Health and HIVIAIDS prevention services in all health care delivery settings in the country, and should include Counselling and Testing at a Reproductive and Child Health services setting". Moreover, recognizing benefits and demand for HIV and SRH services linkages the document captures that "clients going for counselling and testing centre services should also be linked to a wide range of other services including tuberculosis, reproductive health and family planning, social and spiritual support and home based care services". Also, community members, particularly men and other close family members should be educated to play a more active part in 
supporting mothers to access and use PMTCT services and in reducing stigma, denial and discrimination. It further insists a need for postpartum services including family planning and reproductive health information and services to be offered within PMTCT settings or through referral to all women who wish to prevent future unintended pregnancies.

The Guidelines for HIV Testing and Counselling in Clinical Settings (URT/M oHSW/NACP, 2007) has emphasized a need for linking available services in order to minimize the resources to be used as well maximizing services that clients might need. These guidelines provide guidance to health care practitioners as they initiate HIV testing and counselling in the clinical settings. Family planning is one of four priorities for provider initiated testing and counselling (PITC), Posttest counselling recommendations include referral for family planning and family planning group information sessions are highlighted as an opportunity to provide information on HIV counselling and testing.

\section{Service Accessibility according to National Laws, Policies and Guidelines}

\section{Gender Considerations}

In Tanzania, there are no gender biases in relation to policies that mandate services integration. The National Policy on HIV/AIDS, 2001(URT/PMO 2001), accords men and women equal status and opportunities for education, access to reproductive health education, and access to health care services, leadership and advancement in all spheres. Furthermore, cementing the existence of linkages, ministries responsible for youth (regardless of gender differences) in collaboration with local government councils, NGOs, FBOs are required to develop participatory HIVIAIDS and SRH health education for out of school youths. Moreover, the existence of the policy in this regard, demands more vigorous translation to practical activities within communities.

\section{Rights of PLWH/A in relation to HIV/SRH services linkages}

According to the HIV/AIDS Prevention and Control Act of 1998 ( URT, 1998) on health and support services to people living with HIV and AIDS, the government is committed to using available resources to ensure that every person living with HIV and AIDS, vulnerable children and orphans are accorded with basic health services. It further states that PLWH/A have the right to marry and have a family. Further on Stigma and Discrimination, the government act is cognizant and protective of the rights of PLWH/A in that, a person shall not formulate a policy, enact any law or act in a manner that discriminate directly or by its implication persons living with HIV and AIDS, orphans or their families. It is also stated that any health practitioner who deals with HIV and AIDS shall provide health services without any kind of stigma or discrimination and a person shall not stigmatize or discriminate in any manner any other person on the grounds of such person's actual perceived or suspected HIV and AIDS status.

\section{Budgetary Support for linkages}

Sources of funds for both SRH and HIV services are the government, donors and other non government/faith based organizations. However, such funding comes separately i.e. separate funds for HIV and other funds for SRH services. As per Guidelines for the Preparation of Medium Term Expenditure Framework (MTEF) for 2007/08 -2009/10 (URT, 2007), the meagre budget allocation to the health sector has been a hindrance to effective implementation of the Essential RCH Package. During 2005/2006, the health budget allocation was US\$ 3413 million, which is $10.1 \%$ of the total Government budget, below the recommended Abuja target of $15 \%$ Due to other competing health priorities such as malaria, HIV/AIDS and tuberculosis, the budget allocation for reproductive and child health is still limited. According to the MTEF guidelines on priority areas for resource allocation on SRH and HIV services, for the period 2007/2008 and 2009/2010, despite the government emphasis on the implementation of the reproductive and child health services, 
there was no increase in the funds that were allocated.

Generally, the term 'family planning' is mentioned in all HIV policies and guidelines included in the review, there is, however, inconsistent emphasis on family planning content, in which contexts it is referred, and in the recommendations made. There are a few mentions of 'reproductive health or services' in some of the guidelines, but it is unclear whether or not this specifically includes family planning. On the other side, HIV is also mentioned in all FP policy and guideline documents. There is, however as in FP documents, inconsistent emphasis on HIV/AIDS content, in which contexts it is referred, and in the recommendations made. Moreover, the strongest mandate for integrating HIV/AIDS services into family planning and reproductive health is found in the National Policy Guidelines for Reproductive and Child Health Services (2003). The HIV guidelines with the strongest comprehensive elements for family planning service integration are in the National Prevention of Mother to Child Transmission Guidelines of 2003 and 2007" (URT/M oHSW, 2003 and URT/M oHSW 2007). Recommendations for integrated services increased in the PMTCT Guidelines updates, and decreased within the Testing and Counselling Guideline updates. However, there are no direct mentions of or recommendations for HIV/AIDS training for Family Planning/Reproductive Health programme managers or service providers in any of the documents reviewed.

\section{Conclusion}

This review aimed at getting an insight into the state of integration of sexual and reproductive health and HIV services in Tanzania, the results of which would inform policy and strategic considerations for linking the two services. Specifically, the study sought to answer two questions: (i) What is the level and effectiveness of linkages between sexual and reproductive health and HIV related policies, national laws, operational plans and guidelines?; and (ii) To what extent are HIV services integrated into SRH services and SRH services integrated into HIV services?

The review revealed that linkages between sexual and reproductive health and HIV related policies, national laws, operational plans and guidelines are generally evident. The majority of policies and operational guidelines in both the FP and HIV sides mandate bi-directional linkages. All the six SRH documents reviewed had a mention of access to HIV services. On the other hand, of the same number of HIV documents reviewed, five had a mention of access to SRH services. On the level and effectiveness of these linkages, the review looked at the degree with which the policies translated into operational plans and guidelines with a focus on three key interventions/goals: Family Planning as a HIV prevention strategy, FP services as a right for PLWH/A, and universal HIV testing.

SRH services are said to be recognized as a right for PLWHA if, also, the operational policies and service protocols guiding service delivery for PLWHA are in fact aligned or facilitating access to FP by a PLW/A. The assessment review of the National Multi-sector Strategic Framework on HIV/AIDS and the Health Sector Strategy 2008-2012 (PMO/TACAIDS, 2007) show this concept to generally be supported as it advocates a shift from vertical to integrated function of health sectors. Further review of M anagement of HIVIAIDS acknow ledges that family planning as part of a comprehensive package of prevention interventions offered to PLWHA.

Universal HIV testing is a key intervention that is reflected in the HIV strategy. In this case, anyone who should be tested for HIV should have access to these services. Given the fact that SRH clients are very much susceptible to HIV infection, targeting these services for provision of HIV testing is critical. The review of the National Multi-sectoral strategy revealed existence of integrated services. This is further supported by the existence of the PITC guidelines which mandates provision of HIV testing in FP clinics (PM O/TACAIDS, 2007).

The global reproductive health strategy of the World Health Organization (WHO) outlines commitments to attaining goals related to global sexual and reproductive health. These goals 
have five core elements: improving antenatal, delivery, postpartum, and newborn care; providing high-quality FP services, including infertility services, and eliminating unsafe abortion; combating sexually transmitted infections (STIS), including HIV, reproductive tract infections (RTIs), cervical cancer, and other gynecological morbidities; promoting sexual health; and the larger crosscutting issues of gender-based violence, human rights, and male involvement. WHO's strategy emphasizes the need to strengthen sexual and reproductive health services as the basis of HIV treatment and prevention (WHO, 2004).

The Government of Tanzania has introduced provider-initiated with a goal of minimizing resources to be used and maximizing services that clients may need. Indeed, HIV counselling and testing appear to be the main reason for visiting the HIV clinic regardless of gender. Likewise FP services are the major reason for visit in the SRH facilities, although there is gender imbalance.

When it comes to health system support of integration, the assessment relied mainly on responses from the key informants who reported the existence of strategies to lobby for leadership support for integrated services as well as the presence of joint planning mechanism between HIV and SRH departments and/or institutions. Annual planning at the district level is done jointly by the Council Health Management Teams of which managers of the two programmes are members. How ever in the Comprehensive Council Health Plans activities for two services appear separately suggesting that linkages at this level are yet to be fully realized. A joint mechanism at national level is the FP/HIV Technical Working Group which serves as a coordination mechanism mainly between implementing partners.

Financial support and design of programmes also influence the extent of integration. It is clear that funding for FP has dwindled over the years for various reasons, including HIV crisis. On the other hand, whereas HIV programmes have comparably larger amounts of funding, the implementation of interventions is highly verticalised and not facilitating integration of HIV services into other existing services.

In conclusion, this review suggests that the linkage of the two services does exist and its operationalisation is feasible. However, policies and guidelines only specify services to be integrated without due consideration of resources and structural orientation for linked services. Clear policies and guidelines produce the best results. In the case of linkage, an agreement on what are priority areas for linkages, how these should be linked and a collection of feasible experience from the field could provide the appropriate basis.

\section{References}

Abrams, E.J ., Matheson, P.B., Thomas, P.A., Thea, D.M., Krasinski, K., Lambert, G. (1995) Neonatal predictors of infection status and early death among 332 infants at risk of HIV-1 infection monitored prospectively from birth. New York City Perinatal HIV Transmission Collaborative Study Group. Paediatrics 96, 451-458.

Brocklehurst, P. \& French, R. (1998) The association between maternal HIV infection and perinatal outcome: a systematic review of the literature and meta-analysis. British J ournal of Obstetrics and Gynaecology 105, 836-848.

Desgrées-du-Loû, A., M sellati, P., Viho, I., Yao, A., Yapi, D., Kassi, P. (2002) Contraceptive use, protected sexual intercourse and incidence of pregnancies among African HIV-infected women. DITRAME ANRS 049 Project, Abidjan 1995-2000. International J ournal of STD \& AIDS 13, 462-468.

HSHSP, 2008, The Health Sector Strategy for HIV/AIDS, 2008-2012

Homsy, J., Bunnell, R., Moore, D., King, R., Malamba, S., Nakityo, R. (2009) Reproductive intentions and outcomes among women on antiretroviral therapy in rural Uganda: a prospective cohort study. PLoS One 4, e4149. 
HRSH, 2008, The Plan III Human Resources for Health Strategic Plan, 2008-2013

Martin, R., Boyer, P., Hammill, H., Peavy, H., Platzker, A., Settlage, R. (1997) Incidence of premature birth and neonatal respiratory disease in infants of HIV-positive mothers. The Paediatric Pulmonary and Cardiovascular Complications of Vertically Transmitted Human Immunodeficiency Virus Infection Study Group. J ournal of Pediatrics 131, 851-856.

MoHSW (2005) National Standards for Adolescent Friendly Reproductive Health Services, March 2005

MoHSW (2005), The Family Planning Programme Components and Performance Service Standards, 2005

MoHSW (2008) The National Road Map Strategic Plan to Accelerate Reduction of Maternal, Newborn and Child Deaths in Tanzania. Ministry of Health and Social Welfare, 2008 - 2015

MoHSW (2008) Integrating family planning and HIV/AIDS services in Tanzania, a stakeholders consultative report.

MoHSW (2009) Health Sector Strategic Plan III. United Republic of Tanzania: Ministry of Health and Social Welfare, Dar es Salaam, J uly 2009-J une 2015.

NACP (2008), The National Guidelines for M anagement of HIV and AIDS, Third Edition 2008.

PMO/TACAIDS (2003), Prime Minister's Office, Tanzania Commission for HIV/AIDS, Second National Multi-Sectoral Strategic Framew ork on HIV and AIDS, J anuary 2003.

PM 0/TACAIDS (2007), Prime M inister's Office, Tanzania Commission for HIV/AIDS, National M ultisector Strategic Framework on HIV/AIDS and the Health Sector Strategy 2008-2012, second Edition 2007

Reynolds, H.W., Janowitz, B., Wilcher, R. \& Cates, W. (2008) Contraception to prevent HIVpositive births: current contribution and potential cost-savings in PEPFAR countries. Sexually Transmitted Infections 84 (Suppl II), ii49-53.

Rochat, T.J ., Richter, L.M., Doll, H.A., Buthelezi, N.P., Tomkins, A. \& Stein, A. (2006) Depression among pregnant rural South African women undergoing HIV testing. JAMA 295, 13761378.

Sera ya Afya (2007) Sera ya Afya. Wizara ya Afya, J amhuri ya M uungano wa Tanzania.

Stover, J., Fuchs, N., Halperin, D. (2003) Costs and benefits of adding family planning to services to prevent mother-to-child transmission of HIV (PMTCT). Washington, DC: The Futures Group, 2003.

Sweat, M.D., O'Reilly, K.R., Schmid, G.P., Denison, J \& de Zoysa, I. (2004) Cost -effectiveness of nevirapine to prevent mother-to-child HIV transmission in eight African countries. AIDS 18, 1661-1671

URT (1998) Tanzania: HIV and AIDS Prevention and Control Act (1998)

URT, (2007) Guidelines for the Preparation of Medium Term Expenditure Framework (MTEF) for 2007/08 -2009/10

URT/PM O (2001) Prime M inister's Office, National Policy on HIV and AIDS, September 2001

URT/M oHSW (2003) The National Policy Guidelines for Reproductive and Child Health Services

URT/M oHSW (2007) The Comprehensive Family Planning Procedure M anual, March 2007

URT/M oHSW (2007) National Prevention of Mother to Child Transmission Guidelines, 2007

URT/M oHSW (2008) The Plan III Human Resources for Health Strategic Plan, 2008-2013 (J anuary, 2008)

URT/M oHSW/NACP (2007) The Guidelines for HIV Testing and Counselling in Clinical Settings

WHO (2004) Glion consultation on strengthening the linkages between reproductive health and HIV/AIDS: family planning and HIV/AIDS in women and children. 2004. http://www.who.int/reproductive-health/stis/docs/glion_consultationsummary 